\title{
Connected cubic graphs with the maximum number of perfect matchings
}

\author{
Peter Horak \\ University of Washington, Tacoma \\ horak@uw.edu
}

\author{
Dongryul Kim \\ Stanford University \\ dkim04@stanford.edu
}

June 25, 2020

\begin{abstract}
It is proved that for $n \geq 6$, the number of perfect matchings in a simple connected cubic graph on $2 n$ vertices is at most $4 f_{n-1}$, with $f_{n}$ being the $n$-th Fibonacci number. The unique extremal graph is characterized as well.

In addition, it is shown that the number of perfect matchings in any cubic graph $G$ equals the expected value of a random variable defined on all 2-colorings of edges of $G$. Finally, an improved lower bound on the maximum number of cycles in a cubic graph is provided.
\end{abstract}

\section{Contents}

1 Introduction 1

2 The maximal graphs 3

3 Proof of Theorem 1.4 6

4 Proof of Theorem $1.5 \quad 16$

5 Proof of Theorem 1.6 18

Acknowledgment 20

$\begin{array}{ll}\text { References } & 20\end{array}$

\section{Introduction}

It is natural to ask how many cycles, Hamiltonian cycles, and 2-factors a graph can have. To the best of our knowledge this question for cycles was considered 
for the first time by Ahrens [1] in 1897. This paper focuses on the number of 2 -factors in cubic graphs.

We first note that in a cubic graph, the complement of a 1-factor (i.e., a perfect matching) is a 2 -factor, and vice versa. Therefore, for a cubic graph $G$, the number of perfect matchings, the number of 1-factors, the number of 2 -factors are all equal, and we denote this by

$$
\operatorname{PerMat}(G)=\operatorname{Fac}(G) .
$$

There is an extensive literature on the number of perfect matchings in cubic graphs. We mention here only the most pertinent results. It is shown in [6] that the number of perfect matchings in a 2-connected cubic graph is at least exponential with its order, thus confirming an old conjecture of Lovász and Plummer 9 .

As for the maximum number of perfect matchings, Alon and Friedland 3 ] proved a general result. Its restriction to cubic graphs states:

Theorem 1.1 (Alon-Freidland [3], 2008). For a simple cubic graph $G$ on $2 n$ vertices,

$$
\operatorname{PerMat}(G) \leq 6^{n / 3} .
$$

This bound is tight, and it is attained by taking the disjoint union of bipartite complete graphs $K_{3,3}$.

In other words, the above theorem says that the complete bipartite graph $K_{3,3}$ has the highest "density" of perfect matchings among all cubic graphs; thus the disjoint union of its copies constitutes the extremal graph. However, this result does not provide any insight into the structure of extremal connected cubic graphs.

Remark 1.2. When $G$ is bipartite, Theorem 1.1 is an immediate consequence of the Brègman-Minc inequality, proved by Brègman [4] in 1973. The contribution of Alon and Friedland was introducing a clever trick that extended the result from bipartite graphs to all graphs. We later use the same trick, in the form of Lemma 3.6 .

If only connected cubic (but not necessarily simple) graphs are considered then Galbiati [7] proved the following theorem.

Theorem 1.3 (Galbati [7, 1981). For a connected cubic (multi) graph $G$ on $2 n$ vertices,

$$
\operatorname{PerMat}(G) \leq 2^{n}+1 .
$$

This bound is tight, and it is attained by taking a cycle of length $2 n$ and putting parallel edges alternatively.

The main result of this paper characterizes the extremal graphs if only simple connected cubic graphs are taken into account. It is somewhat counter-intuitive that the extremal graphs $M_{n}$ are 2-connected but not 3-connected for large $n$. 
Theorem 1.4. Let $G$ be a simple, connected cubic graph on $2 n$ vertices. Then, $\operatorname{PerMat}(G) \leq m_{n}$, and moreover, equality is achieved if and only if $G$ is isomorphic to the graph $M_{n}$ (see Section 0 for the definition of $m_{n}$ and $M_{n}$ ). In particular, for $n \geq 6$ we have a sharp bound

$$
\operatorname{PerMat}(G) \leq 4 f_{n-1},
$$

where $f_{n-1}$ is the $(n-1)$ th Fibonacci number.

In addition, we derive a formula for counting the number of perfect matchings.

Theorem 1.5. For a cubic graph $G$ of order $2 n$, the number of perfect matchings can be calculated as the expected value $\operatorname{PerMat}(G)=\mathrm{E}(X)$, where $X$ is a random variable defined on the set of all 2-colorings $c$ on the edges of $G$, each coloring equally likely, and $X(c)=(-3)^{m_{c}}$, where $m_{c}$ is the number of vertices of $G$ incident in $c$ with three edges of the same color.

The above formula is not feasible for practical calculations but we believe it is of a theoretical value. The proof is based on interpreting the number of perfect matchings as an evaluation of a suitable quantum field theory. We include only a sketch of the proof in Section 4.

Finally, we turn to the original question of Ahrens [1], on the maximum number of cycles in a connected graph $G=(V, E)$. It is most convenient to study the number of cycles in a connected graph $G$ with respect to its cyclomatic number $r(G)=|E|-|V|+1$, since the number of cycles is bounded by $2^{r(G)}-$ 1. Let $\Psi(r)$ be the maximum number of cycles among all graphs with the cyclomatic number equal to $r$. Entringer and Slater [5] showed that the problem of determining $\Psi(r)$ can be reduced to cubic graphs; they proved that, for $r \geq 3$, there is a connected cubic graph with the cyclomatic number $r$ and $\Psi(r)$ cycles. In addition they conjectured that $\Psi(r) \sim 2^{r-1}$. So far the best upper bound has been provided by Aldred and Thomassen [2] who proved there that $\Psi(r) \leq \frac{15}{16} 2^{r}$.

As for the lower bounds on $\Psi(r)$, the first lower bound $\Psi(r) \geq 2^{r-1}+f(r)$, where the error function $f(r)$ is exponential has been given in [8]. In Section [5] we show that a graph obtained from $C_{n} \times K_{2}$ by replacing consecutive pairs of "parallel" rungs by "crossing" ones provides a bound on $\Psi(r)$ with an improved exponential error term.

Theorem 1.6. There exists a constant $c>0$ for which

$$
\Psi(r) \geq 2^{r-1}+c r 2^{r / 2}
$$

for all sufficiently large $r$.

For a more precise bound, we refer the reader to Section 5 .

\section{The maximal graphs}

Before we prove Theorem [1.4, we collect properties that we will later use to prove Theorem 1.4. The results in this section mostly follow from a routine verification, hence we leave some of the details to the reader. 
We define the Fibonacci numbers by

$$
f_{n}=\frac{1}{\sqrt{5}}\left(\varphi^{n}-\varphi^{-n}\right), \quad \varphi=\frac{1+\sqrt{5}}{2},
$$

so that they satisfy

$$
f_{0}=0, \quad f_{1}=1, \quad f_{2}=1, \quad f_{n}=f_{n-1}+f_{n-2}
$$

for all integers $n$.

For the purpose of stating and proving Theorem 1.4 we define $m_{n}$ for $n \geq 3$ as

$$
m_{2}=3, \quad m_{3}=6, \quad m_{4}=9, \quad m_{5}=13, \quad m_{n}=4 f_{n-1} \text { for } n \geq 6 .
$$

We collect the inequalities among $m_{n}$ that we will use later. Note that we asymptotically have $m_{n} \sim c \varphi^{n}$ where $c \approx 1.106$ and $\varphi \approx 1.618$ is the golden ratio; this is useful for doing a quick sanity check.

\section{Lemma 2.1.}

(i) For $n \geq 8$, we have $m_{n}=m_{n-1}+m_{n-2}$.

(ii) For $n \geq 6$, we have $\frac{3}{2} m_{n-1}<m_{n}$.

(iii) For $n \geq 5$, we have $2 m_{n-2}<m_{n}$.

(iv) For $n \geq 8$, we have $4 m_{n-3} \leq m_{n}$, with equality only for $n=8$.

(v) For $n \geq 9$, we have $6 m_{n-4}<m_{n}$.

(vi) For $n \geq 6$, we have $m_{n+1}<\sqrt{3} m_{n}$.

(vii) For $n \geq 3$, we have $m_{2 n}<m_{n}^{2}$.

(viii) For $a, b \geq 3$, if $a+b \geq 8$ then $m_{a} m_{b}<m_{a+b+1}$.

Proof. (i) follows from the definition that $m_{n}=4 f_{n-1}$ for $n \geq 6$. For (ii)] we can check the inequality by hand when $6 \leq n \leq 8$. When $n \geq 9$, we use induction; assuming that the inequality holds for smaller $n \geq 6$, we can write

$$
\frac{3}{2} m_{n-1}=\frac{3}{2} m_{n-2}+\frac{3}{2} m_{n-3}<m_{n-1}+m_{n-2}=m_{n}
$$

by (i)] since $n \geq 9$. The other inequalities (iii), (iv) (v), (vi) can be proved using a similar inductive argument.

For (vii), we first do the case $n \leq 5$ manually. For $n \geq 6$, we note that

$$
m_{2 n}=4 f_{2 n-1}=4\left(f_{n}^{2}+f_{n-1}^{2}\right)=\frac{1}{4}\left(m_{n+1}^{2}+m_{n}^{2}\right)<\frac{1}{4}\left(\left(\sqrt{3} m_{n}\right)^{2}+m_{n}^{2}\right)=m_{n}^{2}
$$

by (vi). Here, the identity $f_{2 n-1}=f_{n}^{2}+f_{n-1}^{2}$ is folklore.

For (viii) we induct on $a+b$. When $\max (a, b) \leq 7$, we can check manually. If $\max (a, b) \geq 8$, without loss of generally assume that $a \geq 8$. Then by (i) and the inductive hypothesis,

$$
m_{a} m_{b}=m_{a-1} m_{b}+m_{a-2} m_{b}<m_{a+b}+m_{a+b-1}=m_{a+b+1} .
$$

Here, the induction hypothesis applies because $(a-2)+b \geq 8-2+3=9$. 


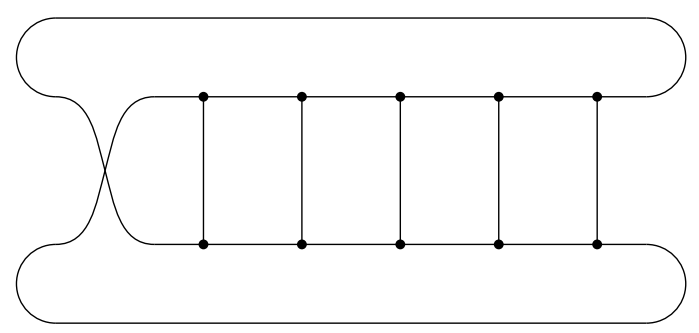

Figure 1: The graph $M_{5}$

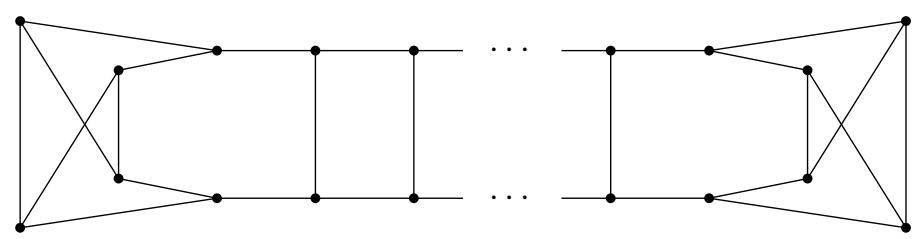

Figure 2: The graph $M_{n}$ for $n \geq 6$

We now define the graphs that attain the maximal number of 2-factors for a given number of vertices.

Definition 2.2. For $n \geq 2$, we define the simple connected cubic graph $M_{n}$ as follows:

- $M_{2}=K_{4}$

- $M_{3}=K_{3,3}$;

- $M_{4}$ is $K_{4,4}$ with a perfect matching removed;

- $M_{5}$ is the Möbius ladder on 10 vertices, see Figure 1

- for $n \geq 6, M_{n}$ is the ladder graph with $K_{3,3}$ inserted at both ends, see Figure 2

Proposition 2.3. For all $n \geq 2$, we have $\operatorname{Fac}\left(M_{n}\right)=m_{n}$.

Proof. For $n \leq 7$, we can count the number of 2-factors explicitly, and check that the number of 2-factors of $M_{n}$ is exactly $m_{n}$. For $n \geq 8$, we induct on $n$. It is shown in Section 3, proof of Theorem 1.4, Subcase 1-1, that

$$
\operatorname{Fac}\left(M_{n}\right)=\operatorname{Fac}\left(M_{n-1}\right)+\operatorname{Fac}\left(M_{n-2}\right) .
$$

Then it follows from Lemma 2.1, (i) that $\operatorname{Fac}\left(M_{n}\right)=m_{n-1}+m_{n-2}=m_{n}$ by the inductive hypothesis. 


\section{Proof of Theorem 1.4}

For the reader's convenience, the proof of Theorem 1.4 will be presented in terms of 2 -factors instead of perfect matchings. We recall that the complement of a perfect matching in a cubic graph is 2 -factor.

The general strategy for proving Theorem 1.4 is to first prove the theorem for bipartite graphs. Then using a trick of Alon and Friedland [3], we deduce the general case. We start with an auxiliary result.

Lemma 3.1. Let $G$ be a simple connected bipartite cubic graph.

(i) The graph $G$ does not have a bridge.

(ii) For every edge $x y$ of $G$, the induced subgraph on $V(G)-\{x, y\}$ has at most two components.

Proof. (i) If it has a bridge, we may remove the edge and take a component of the resulting graph. This is a bipartite graph with one vertex of degree 2 and all other vertices having degree 3 . Counting the number of edges, we see that it is both a multiple of 3 and equal to 2 modulo 3, arriving at a contradiction.

(ii) Denote by $G^{\prime}$ the induced subgraph on $V(G) \backslash\{x, y\}$ and assume that $G^{\prime}$ has at least three components. Let the neighbors of $x$ be $y, a, b$ and the neighbors of $y$ be $x, c, d$. Because $G$ was connected, each one of the components of $G^{\prime}$ contains at least one of the four vertices $a, b, c, d$. By part (i), the edge $x y$ is not a bridge in $G$. This means that there is a path in $G^{\prime}$ connecting either $a$ or $b$ to either $c$ or $d$. Hence, without loss of generality, we may as well assume that $a, c$ are in the same component in $G^{\prime}$. Then $b$ is in a different component than $a, c, d$ in $G^{\prime}$, which means that the edge $x b$ must be a bridge in $G$. This contradicts part (i).

Definition 3.2. For a simple connected bipartite cubic graph $G$, we say that an edge $e=u v$ is a ladder-bridge if the induced subgraph on $V(G) \backslash\{u, v\}$ is disconnected, or equivalently, has two components.

We first prove Theorem 1.4 for bipartite graphs.

Theorem 3.3. Let $G$ be a simple connected cubic bipartite graph on $2 n$ vertices (so automatically $n \geq 3$ ). Then $\operatorname{PerMat}(G)=\operatorname{Fac}(G) \leq m_{n}$, and moreover, equality is achieved if and only if $G$ is isomorphic to the graph $M_{n}$.

Remark 3.4. For $n \leq 8$, we content ourselves with using a computer to verify the statement. (There are 60 simple connected bipartite cubic graphs with at most 16 vertices in total.) However, it is possible to avoid using a computer at all. We can get away with verifying the statement by hand for $n \leq 6$ only (in total, there are only 9 simple connected bipartite graphs with at most 12 vertices), but then the proof of the theorem becomes quite longer as many small cases for $n=7,8$ have to be considered separately. 


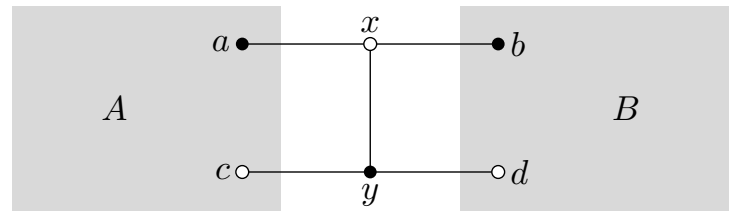

Figure 3: Case 1-a connected bipartite cubic graph $G$ with ladder-bridge $x y$

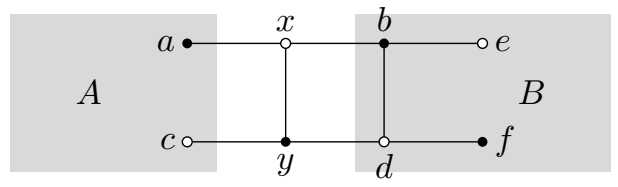

Figure 4: Subcase 1-1-when $x y$ is next to another ladder-bridge $b d$

Proof of Theorem 3.3. We use induction on $n$. For $n \leq 8$, we can perform a computer search on all the cubic bipartite graphs. Hence, let us assume that $n \geq 9$ and that the statement holds for all smaller $n$.

Case 1. The graph $G$ has a ladder-bridge $x y$. Let us write $V(G) \backslash\{x, y\}=$ $A \cup B$, where $A$ and $B$ are the components. Note that if all neighbors of $x$ are in $A \cup\{y\}$, then one of the edges adjacent to $y$ is a bridge of $G$, contradicting Lemma 3.1. Hence one of the neighbors of $x$ is in $A$, one of them is in $B$, and similarly for $y$. Define $a, c \in A$ and $b, d \in B$ so that the neighbors of $x$ are $y, a, b$ and the neighbors of $y$ are $x, c, d$, as in Figure 3 ,

Subcase 1-1. Suppose either $a$ and $c$ are connected by an edge or $b$ and $d$ are connected by an edge. Without loss of generality, we assume that $b, d$ are connected by an edge. Let the neighbors of $b$ be $x, d, e$, and the neighbors of $d$ be $y, b, f$, as in Figure 4. We consider two other graphs: $G^{\prime}$, which is the graph obtained by removing the vertices $x, y$ and connecting $a d, c b$, and $G^{\prime \prime}$, which is the graph obtained by removing vertices $x, y, b, d$ and connecting $a e, c f$, see Figure 5. Note that both $G^{\prime}, G^{\prime \prime}$ are again simple connected bipartite cubic graphs, where connectivity of $G^{\prime \prime}$ follows from the fact that there is a path in $A$ connecting $a, c$. Any 2-factor of $G$ will either contain both $a x, c y$ or contain neither, because these are the only edges connecting a vertex in $A$ and a vertex not in $A$. Using this, we see that there are five possible shapes a 2 -factor of $G$ can take on the induced subgraph on $\{a, b, c, d, e, f, x, y\}$, listed on the leftmost column of Figure 5. Similarly, we can list all the possible shapes a 2-factor of $G^{\prime}$ or $G^{\prime \prime}$ can have on the induced subgraph of $\{a, b, c, d, e, f\}$ or $\{a, c, e, f\}$, and these are depicted on the rightmost column of Figure 5 .

We now modify the 2-factor of $G$ to either a 2-factor of $G^{\prime}$ or a 2-factor of $G^{\prime \prime}$, by simply removing all the edges between $a, b, c, d, e, f, x, y$ and filling in that part with an appropriate diagram. If we do this process by taking the $i$ th diagram on the leftmost column of Figure 5 replacing with the $i$ th diagram on the rightmost column of Figure 5. The process is reversible, as we can similarly 

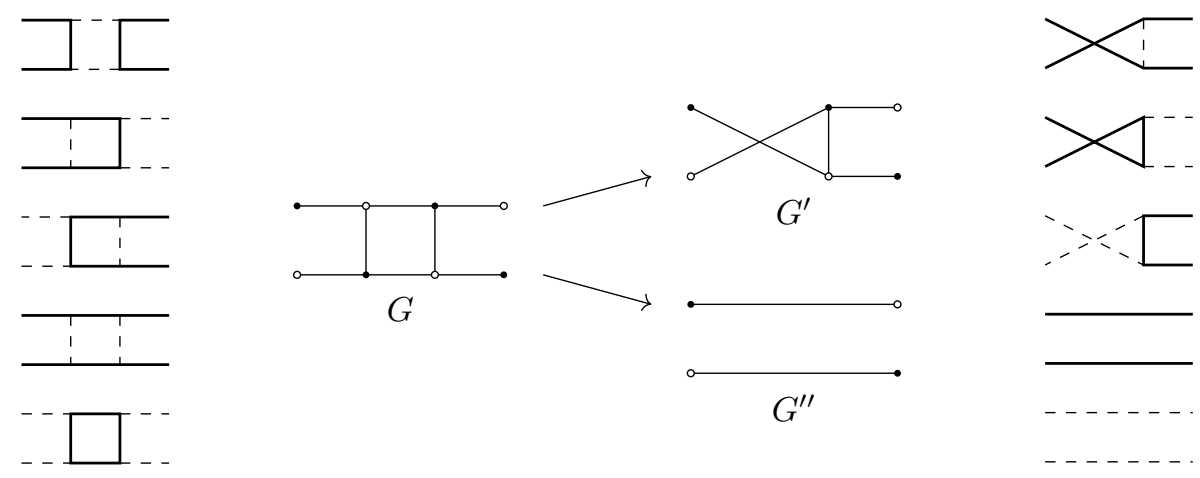

Figure 5: Subcase 1-1-modifying the graph $G$ to $G^{\prime}$ and $G^{\prime \prime}$

take the right hand side diagram and replace it with the left hand side diagram. This shows that

$$
\operatorname{Fac}(G)=\operatorname{Fac}\left(G^{\prime}\right)+\operatorname{Fac}\left(G^{\prime \prime}\right) .
$$

The inductive hypothesis applies to both $G^{\prime}$ and $G^{\prime \prime}$, as they are simple connected bipartite cubic graphs with number of vertices less than $2 n$. Therefore

$$
\operatorname{Fac}(G)=\operatorname{Fac}\left(G^{\prime}\right)+\operatorname{Fac}\left(G^{\prime \prime}\right) \leq m_{n-1}+m_{n-2}=m_{n},
$$

where the last equality holds by Lemma 2.1 (i), since $n \geq 9$.

When does equality hold? Again by the inductive hypothesis, equality holds if and only if $G^{\prime}$ is isomorphic to the graph $M_{n-1}$ and $G^{\prime \prime}$ is isomorphic to the graph $M_{n-2}$. In $G^{\prime}$, the edge $b d$ is a ladder-bridge. To recover $G$ from $G^{\prime}$, we need to insert another ladder-bridge, and it is easy to verify that the resulting graph is always isomorphic to $M_{n}$.

Subcase 1-2. Suppose now that there is no edge between $a, c$ and also between $b, d$. This time, we modify the graph $G$ to $G^{\prime}$ by removing the vertices $x, y$ and then connecting $a, c$ and $b, d$, see Figure 6 Then $G^{\prime}$ is a simple bipartite cubic graph, even though it is not connected. Using the same process of replacing the $i$ th configuraiton of the leftmost column with the $i$ th configuration of the rightmost column, from each 2-factor of $G$ we get a 2 -factor of $G^{\prime}$. Moreover, it is clear that distinct 2-factors of $G$ give distinct 2-factors of $G^{\prime}$, even though some 2 -factors of $G^{\prime}$ do not appear by this process. Therefore $\operatorname{Fac}(G) \leq \operatorname{Fac}\left(G^{\prime}\right)$. On the other hand, $G^{\prime}$ as two components, say $G^{\prime}=G_{1}^{\prime} \cup G_{2}^{\prime}$, where both $G_{1}^{\prime}, G_{2}^{\prime}$ are simple connected bipartite cubic graphs. Then the inductive hypothesis applies, so

$$
\operatorname{Fac}(G) \leq \operatorname{Fac}\left(G^{\prime}\right)=\operatorname{Fac}\left(G_{1}^{\prime}\right) \operatorname{Fac}\left(G_{2}^{\prime}\right) \leq m_{a} m_{n-a-1}
$$

where $G_{1}^{\prime}$ has $2 a$ vertices and $G_{2}^{\prime}$ has $2(n-a-1)$ vertices. Because $n \geq 9$, from Lemma 2.1, (viii), we obtain

$$
\operatorname{Fac}(G) \leq m_{a} m_{n-a-1}<m_{n} .
$$




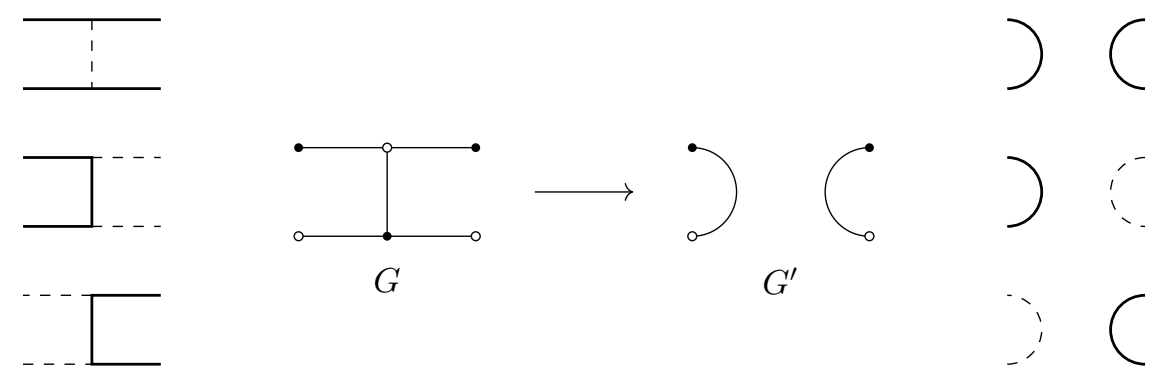

Figure 6: Subcase 1-2-modifying the graph $G$ to $G^{\prime}$

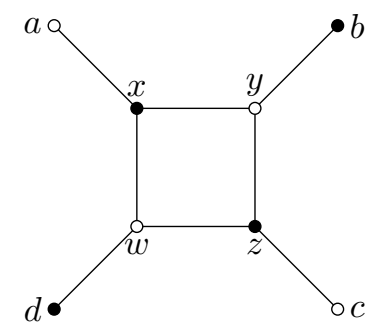

Figure 7: Case 2-a cubic bipartite $G$ with a 4-cycle $x y z w$

Case 2. Now suppose there exists a 4-cycle $x y z w$ in $G$. Denote by $a, y, w$ the neighbors of $x$, by $b, x, z$ the neighbors of $y$, by $c, y, w$ the neighbors of $z$, and by $d, x, z$ the neighbors of $w$, see Figure 7 . By definition, $x, y, z, w$ are all distinct points, but there is no reason for $a, b, c, d$ to be all distinct. It is possible that $a=c$ or $b=d$ or both.

Subcase 2-1. First consider the case when $a=c$ and $b=d$. Now the vertices $a, b, x, y, z, w$ are all distinct. Moreover, $a$ cannot be connected to $b$ by an edge, otherwise we would have $V(G)=\{a, b, x, y, z, w\}$ and $G=K_{3,3}$, contradicting our assumption that $n \geq 9$. Denote by $x, z, e$ the neighbors of $a$ and $y, w, f$ the neighbors of $f$, see Figure 8 . As $a$ and $b$ are not neighbors, we see that all the vertices $a, b, e, f, x, y, z, w$ are distinct. If $e$ and $f$ are connected, then $e f$ becomes a ladder-bridge of $G$, hence this case is already covered in Case 1. Therefore we assume without loss of generality that $e$ and $f$ are not connected by an edge.

Given such a graph $G$, we define a new graph $G^{\prime}$ by removing the vertices $a, b, x, y, z, w$ and then connecting $e$ and $f$. The resulting graph is simple, as $e$ and $f$ were not already connected, and also connected cubic bipartite. We again list the possible configurations of 2 -factors of $G$ restricted to this portion, of which there are 6 as listed on the leftmost column of Figure 9. We may again use the process of replacing the configurations of the leftmost column by configurations of the rightmost column. This time, the process is not injective, 


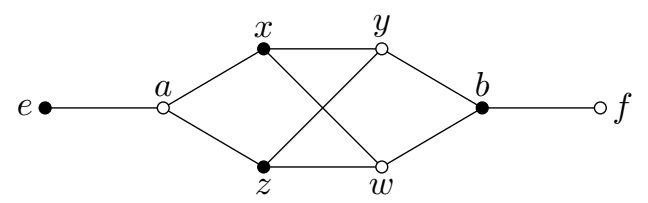

Figure 8: Subcase 2-1—when $a=c$ and $b=d$

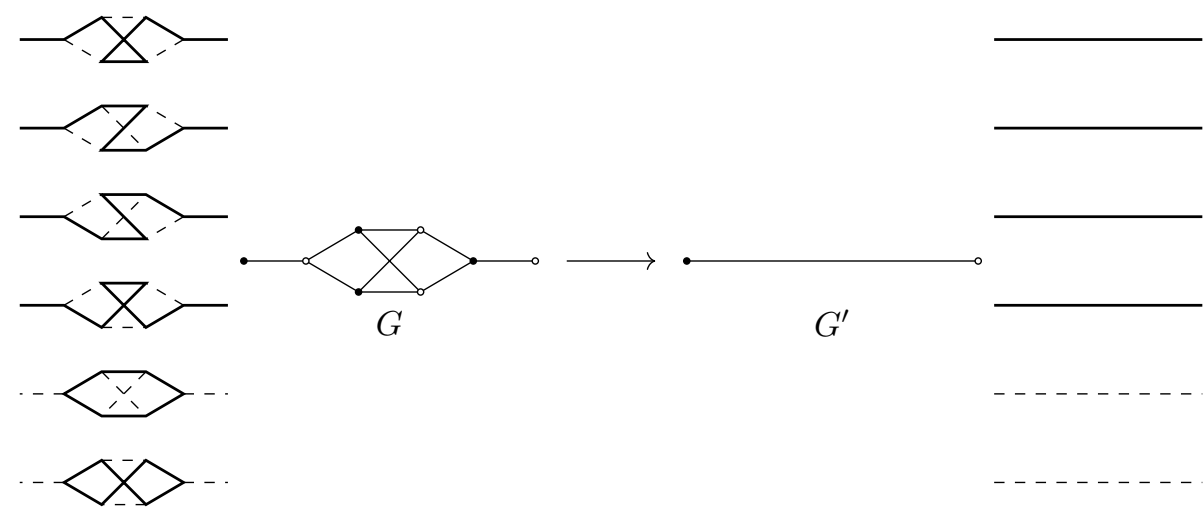

Figure 9: Subcase 2-1-modifying the graph $G$ to $G^{\prime}$

but every 2 -factor of $G^{\prime}$ can occur in at most 4 different ways. This shows that

$$
\operatorname{Fac}(G) \leq 4 \operatorname{Fac}\left(G^{\prime}\right) \leq 4 m_{n-3}<m_{n}
$$

by Lemma 2.1, (iv)

Subcase 2-2. Now suppose that only one of the equalities from $a=c$ and $b=d$ hold. Without loss of generality, assume that $a=c$ and $b \neq d$. Denote by $x, z, e$ the neighbors of $a$, see Figure 10 It is clear that $a, x, y, z, w$ are all distinct, and also distinct from $b, d, e$. The only possible equalities between the points $a, b, d, e, x, y, z, w$ are $d=e$ or $b=e$. But if any of these equalities holds, we are reduced to Subcase 2-1. For instance, if $b=e$ then axyz becomes a 4 -cycle satisfying the assumptions of Subcase 2-1. Therefore we may as well assume that all the vertices $a, b, d, e, x, y, z, w$ are distinct.

We now construct a new graph $G^{\prime}$ by deleting the vertices $x, y, z, w$ and then connecting $a$ with $b$ and $d$. As $b, d, e$ are distinct points, the resulting graph is simple. That is, $G^{\prime}$ is a simple connected bipartite cubic graph, hence satisfies the induction hypothesis. We list the possible 2 -factors of $G$ and consider the replacing process as given by Figure 11. We observe that each 2-factor of $G^{\prime}$ can occur in exactly 2 ways, and therefore

$$
\operatorname{Fac}(G)=2 \operatorname{Fac}\left(G^{\prime}\right) \leq 2 m_{n-2}<m_{n}
$$

by Lemma 2.1, (iii). 


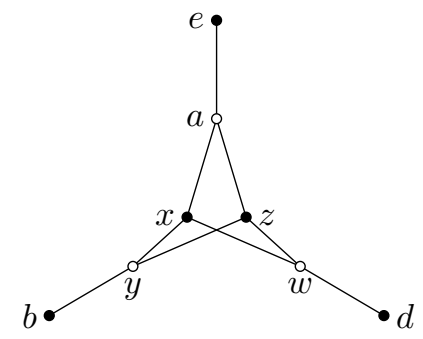

Figure 10: Subcase 2-2-when $a=c$



Figure 11: Subcase 2-2-modifying the graph $G$ to $G^{\prime}$

Subcase 2-3. We are now left with the case when $a \neq c$ and $b \neq d$. In this Subcase, assume that $\{a b, c d\} \cap E(G) \neq \emptyset$ and $\{b c, a d\} \cap E(G) \neq \emptyset$. Then without loss of generality, we can assume that $a$ is connected to both $b$ and $d$ by edges. Denote by $e, a, y$ the neighbors of $b$ and by $f, a, w$ the neighbors of $d$. The vertices $c, e, f$ cannot be all equal, because then we would have $V(G)=\{a, b, c, d, x, y, z, w\}$ which contradicts $n \geq 9$. Thus either the three vertices $c, e, f$ are all distinct, or two of them are equal and distinct from the last one.

Subsubcase 2-3-1. We first assume that two of $c, e, f$ are equal, but not all of them coincide. Here, by symmetry of Figure 12, we may as well assume that $e=f$ but $c \neq e$. Let us denote by $g, b, d$ the neighbors of $e=f$. Then all the 10 vertices $a, b, c, d, e, g, x, y, z, w$ are distinct. We now use the exact same strategy as Subcase 2-1. If $g$ and $c$ are connected by an edge, then the edge $c g$ becomes a ladder-bridge of $G$, hence we can deal with it using Case 1. If $g$ and $c$ are not connected by an edge, consider the graph $G^{\prime}$ obtained from $G$ by deleting the vertices $a, b, d, e, x, y, z, w$ and connecting $c$ and $g$ by an edge. Then $G^{\prime}$ is a simple connected bipartite cubic graph, hence the inductive hypothesis 


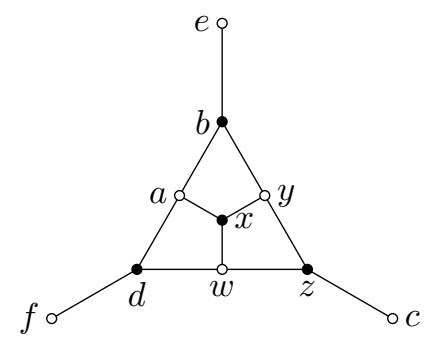

Figure 12: Subcase 2-3-when $a \neq c$ and $b \neq d$ but $a$ is connected to $b, d$
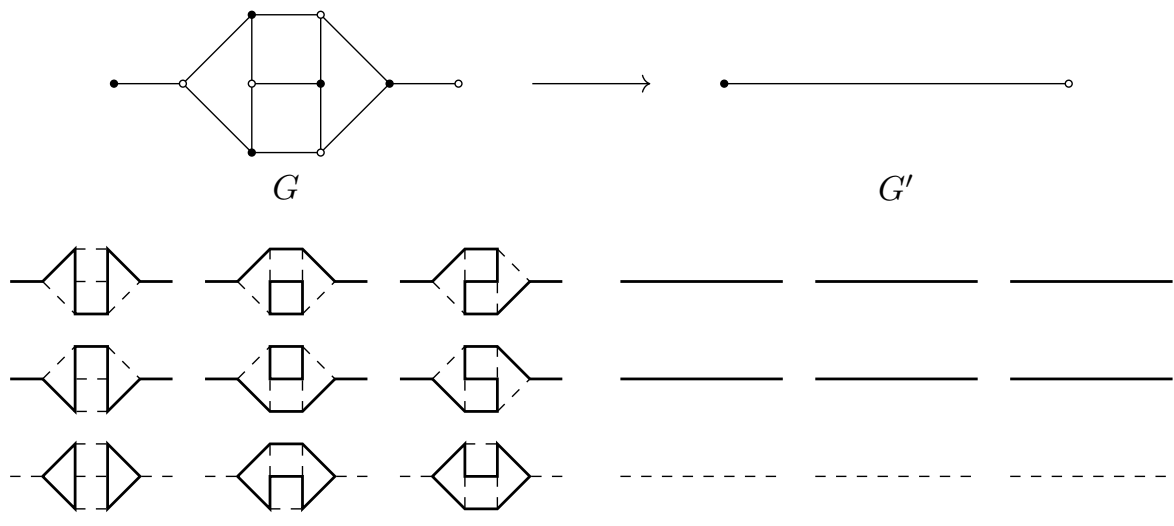

Figure 13: Subsubcase 2-3-1-modifying the graph $G$ to $G^{\prime}$

applies. From Figure 13, we see that

$$
\operatorname{Fac}(G) \leq 6 \operatorname{Fac}\left(G^{\prime}\right) \leq 6 m_{n-4}<m_{n}
$$

by Lemma 2.1, (v)

Subsubcase 2-3-2. We now suppose that $c, e, f$ are all distinct. This time we follow Subcase 2-2. Consider the graph $G^{\prime}$ obtained by deleting the vertices $a, d, x, y, z, w$ from $G$ and the connecting $b$ to both $c, f$. Since $c, e, f$ are distinct points, the modified graph $G^{\prime}$ is simple, and also connected bipartite cubic. We can list the possible 2-factors of $G$ restricted to the subgraph as in Figure 14 . Now every 2 -factor of $G^{\prime}$ can be obtained from a 2 -factor of $G$ in exactly 3 ways, hence

$$
\operatorname{Fac}(G)=3 \operatorname{Fac}\left(G^{\prime}\right) \leq 3 m_{n-3}<4 m_{n-3}<m_{n}
$$

by Lemma 2.1, (iv)

Subcase 2-4. Finally, we assume that $a \neq c, b \neq d$, and either $\{a b, c d\} \cap$ $E(G)=\emptyset$, or $\{b c, a d\} \cap E(G)=\emptyset$. Without loss of generality, suppose that $a, b$ are not connected by an edge and $c, d$ are also not connected by an edge. In this case, as in Subcase 1-1, we consider two graphs. Let $G^{\prime}$ be the graph obtained from $G$ by removing $x, w$ and then connecting $a, z$ and $y, d$. Let $G^{\prime \prime}$ 

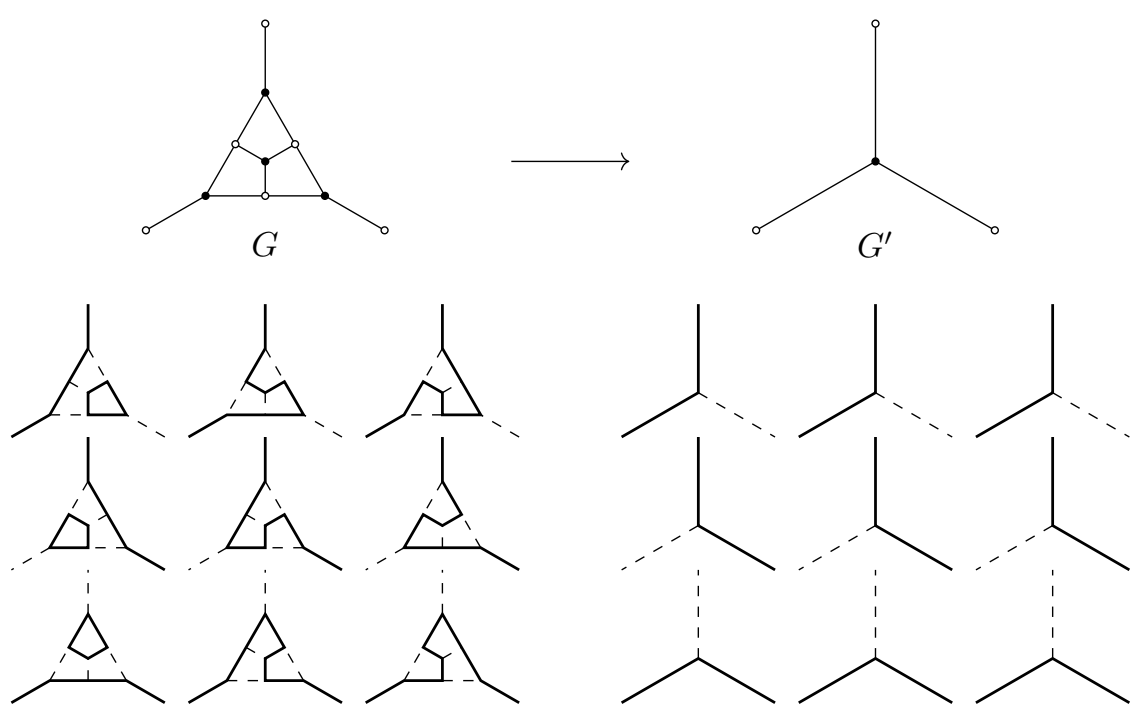

Figure 14: Subsubcase 2-3-2-modifying the graph $G$ to $G^{\prime}$

be the graph obtained from $G$ by removing $x, y, z, w$ and then connecting $a, b$ and $c, d$. Since we have assumed that $a, b$ and $c, d$ are not already connected in $G$, we see that $G^{\prime}, G^{\prime \prime}$ are both simple graphs. It is clear that $G^{\prime}$ is connected. We may assume that $G^{\prime \prime}$ is also connected, because if it is not connected then removing $x, y$ from $G$ disconnects the graph. This would mean that $x y$ is a ladder-bridge of $G$, but then Case 1 handles the situation. Therefore we may suppose both $G^{\prime}$ and $G^{\prime \prime}$ are simple connected bipartite cubic graphs, and the inductive hypothesis applies. We now enumerate the posssible 2-factors and do the replacement procedure according to Figure [15. From the usual analysis, it follows that

$$
\operatorname{Fac}(G) \leq \operatorname{Fac}\left(G^{\prime}\right)+\operatorname{Fac}\left(G^{\prime \prime}\right) \leq m_{n-1}+m_{n-2}=m_{n}
$$

by Lemma 2.1, (i).

We now analyze the equality case. Similarly to Subcase 1-1, by the inductive hypothesis, equality holds only if $G^{\prime}$ and $G^{\prime \prime}$ are isomorphic to $M_{n-1}$ and $M_{n-2}$. For the equality $\operatorname{Fac}(G)=\operatorname{Fac}\left(G^{\prime}\right)+\operatorname{Fac}\left(G^{\prime \prime}\right)$ to holds, we further need that there is no 2-factor of $G^{\prime}$ using the edges $a z, z y, y b$ and also no 2-factor using the edges $d y, y z, z c$. By inspection, we see that the only edges $e$ in $M_{n-1}$ satisfying the above property for $y z$ are precisely the ladder-bridges. This shows that $y z$ corresponds to a ladder-bridge in $M_{n-1}$, and modifying $G^{\prime}$ to $G$ shows that $G$ is isomorphic to the graph $M_{n}$.

Case 3. Since Case 2 was when $G$ has a 4 -cycle, we now assume that $G$ has no 4 -cycles. Since $G$ is bipartite, this implies that all cycles of $G$ has length at least 6 . Pick an arbitrary vertex $x$, denote its neighbors by $y, z, w$, denote the neighbors of $y$ by $x, a, b$, the neighbors of $z$ by $x, c, d$, and the neighbors of 


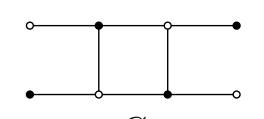

G

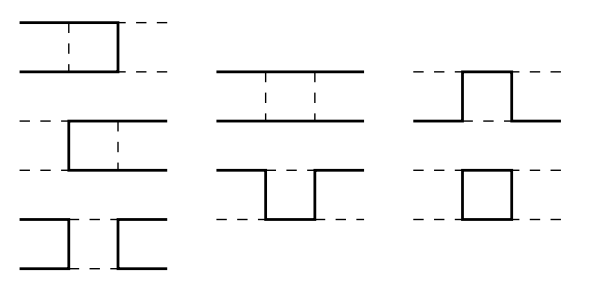





Figure 15: Subcase 2-4-modifying the graph $G$ to $G^{\prime}$ and $G^{\prime \prime}$

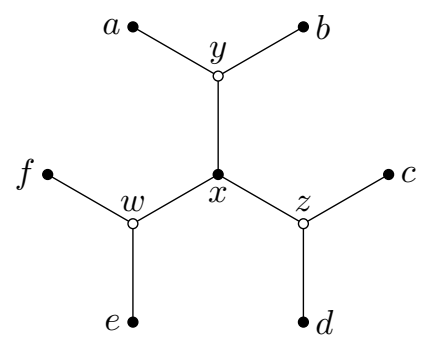

Figure 16: Case 3-when there is no 4-cycle

$w$ by $x, e, f$, as in Figure 16 . As $G$ has no cycles of length smaller than 6 , we immediately see that the vertices $x, y, z, w, a, b, c, d, e, f$ are all distinct.

We now consider six graphs constructed from $G$. The graph $G_{j}^{y}$ for $j=1,2$ are obtained from $G$ by removing the vertices $x, y$, and then adding the edges $a w$ and $b z$ for $j=1$, and adding $a z$ and $b w$ for $j=2$. The other graphs $G_{1}^{y}, G_{2}^{y}, G_{1}^{z}, G_{2}^{z}$ are constructed similarly, as depicted in Figure 17. It is clear that the graphs $G_{j}^{y}, G_{j}^{z}, G_{j}^{w}$ are all simple bipartite cubic graphs. We claim that we may assume that these graphs are also connected for $1 \leq i \leq 6$. For instance, suppose that $G_{1}^{y}$ is not connected. Then $G_{1}^{y}$ with the edges $a w$ and $b z$ removed is also not connected. On the other hand, this graph is what we get when we remove the two vertices $x, y$ from $G$. Thus $G_{1}^{y}$ not being connected implies that $x y$ is a ladder-bridge in $G$. Since this is already covered in Case 1, we may as well assume that $G_{1}^{y}$ is connected. A similar argument works for all other graphs, hence we may assume that the modified graphs are all simple connected bipartite cubic graphs. Hence the inductive hypothesis holds for every $G_{j}^{y}, G_{j}^{z}, G_{j}^{w}$.

Now we claim that given any 2 -factor of $G$, it can be modified to a 2 -factor of four of the graphs $G_{j}^{v}$ (with $v \in\{y, z, w\}$ ), in such a way that the 2-factor of $G$ can be recovered from any of the modified 2-factors. By symmetry, we only need to consider the case when the 2 -factor on the induced subgraph on 

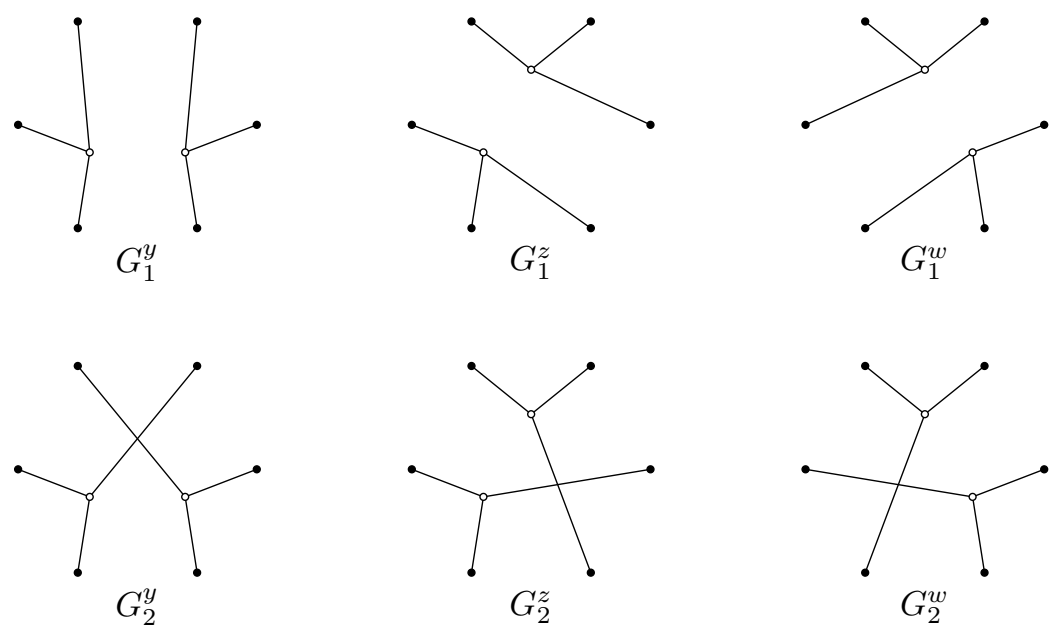

Figure 17: Case 3 -modifying the graph $G$ to $G_{j}^{y}, G_{j}^{z}, G_{j}^{w}$

$\{x, y, z, w, a, b, c, d, e, f\}$ is as in Figure 18. In this case, the 2-factor of $G$ can be turned into 2-factors of $G_{1}^{z}, G_{1}^{w}, G_{2}^{y}, G_{2}^{w}$ without changing it outside the depicted region. This shows that

$$
4 \operatorname{Fac}(G) \leq \sum_{v, j} \operatorname{Fac}\left(G_{j}^{v}\right) \leq 6 m_{n-1}
$$

and by Lemma 2.1 (ii) we have $6 m_{n-1}<4 m_{n}$. This concludes the proof.

Let us now deduce Theorem 1.4 from Theorem 3.3. What we need is a clever lemma of Alon and Friedland [3].

Definition 3.5. Let $G$ be a simple cubic graph. Define a new graph $D(G)$ with vertices

$$
V(D(G))=V(G) \times\{1,2\}
$$

and edges

$$
E(D(G))=\{(v, 1)(w, 2): v w \in E(G)\} .
$$

Note that $D(G)$ is always a simple cubic bipartite graph if $G$ is a simple cubic graph. Moreover, if $G$ is connected and not bipartite, then $D(G)$ is connected.

Lemma 3.6 (Alon-Friedland [3]). Let $G$ be a simple cubic graph. Then

$$
\operatorname{Fac}(G)^{2} \leq \operatorname{Fac}(D(G))
$$

We can finally prove Theorem 1.4 .

Proof of Theorem 1.4. For $n=2$, there is only one simple cubic graph on 4 vertices, namely $K_{4}$. Hence there is nothing to prove. 


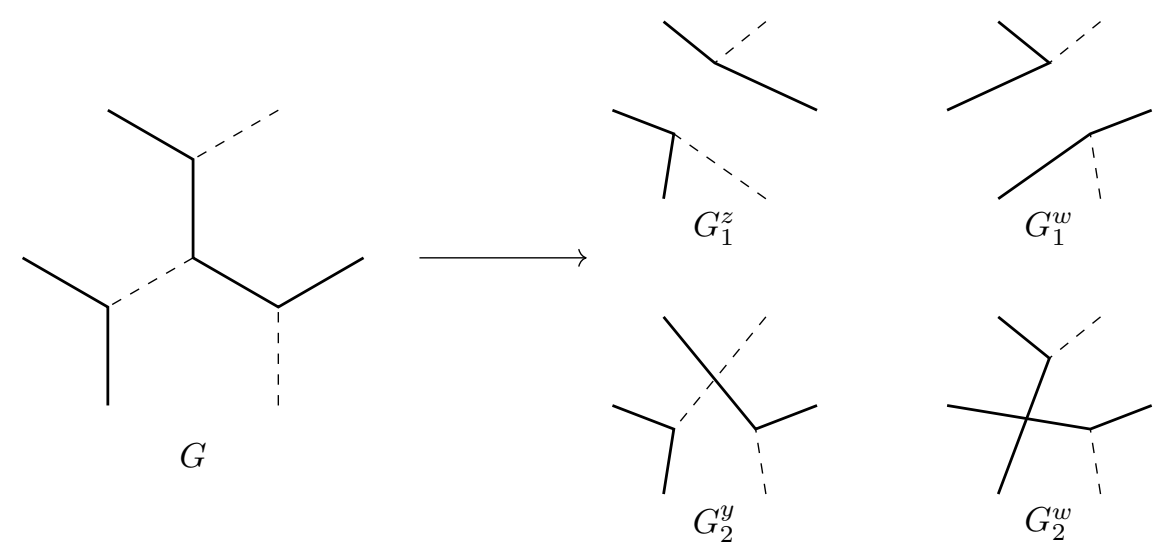

Figure 18: Case 3-modifying a 2-factor of $G$ to 2-factors of $G_{1}^{z}, G_{1}^{w}, G_{2}^{y}, G_{2}^{w}$

We now assume that $n \geq 3$. If $G$ is not bipartite, then $D(G)$ is connected and bipartite, so

$$
\operatorname{Fac}(G)^{2} \leq \operatorname{Fac}(D(G)) \leq m_{2 n}<m_{n}^{2}
$$

by Lemma 3.6. Theorem 3.3 and Lemma 2.1, (vii). This shows that $\operatorname{Fac}(G)<$ $m_{n}$ if $G$ is not bipartite. The remaining case is when $G$ is bipartite, and this is covered by Theorem 3.3 .

\section{Proof of Theorem 1.5}

Due to the connection with quantum physics, we use the bra-ket notation for denoting vectors. Consider the 2-dimensional real vector space

$$
B=\mathbb{R}|0\rangle \oplus \mathbb{R}|1\rangle=\{a|0\rangle+b|1\rangle: a, b \in \mathbb{R}\}
$$

with basis $|0\rangle$ and $|1\rangle$. Its $n$-fold tensor product $B^{\otimes n}=B \otimes \cdots \otimes B$ is a $2^{n}$-dimensional real vector space with basis

$$
\left|s_{1}\right\rangle \otimes\left|s_{2}\right\rangle \otimes \cdots \otimes\left|s_{n}\right\rangle
$$

where $s_{1}, s_{2}, \ldots, s_{n} \in\{0,1\}$.

Consider the element

$$
\alpha=|0\rangle \otimes|0\rangle+|1\rangle \otimes|1\rangle \in B \otimes B
$$

and the linear map $\beta$ is given by

$$
\begin{aligned}
\beta: B \otimes B \otimes B & \rightarrow \mathbb{R}, \\
\left|s_{1}\right\rangle \otimes\left|s_{2}\right\rangle \otimes\left|s_{3}\right\rangle & \mapsto \begin{cases}1 & \text { there are exactly two } 0 \text { among } s_{1}, s_{2}, s_{3}, \\
0 & \text { otherwise. }\end{cases}
\end{aligned}
$$


Given a cubic graph $G=(V, E)$ on $2 n$ vertices, consider its incidence set

$$
\Phi=\{(v, e) \text { : vertex } v \text { is incident to edge } e\} \subseteq V \times E .
$$

For each edge $e=u v \in E$, we have $(u, e),(v, e) \in \Phi$. To $e$ we assign $\alpha \in B \otimes B$, where we interpret the two factors of $B$ as corresponding to $(u, e)$ and $(v, e)$ each, and take their tensor product over all $e$. The resulting element

$$
\alpha^{\otimes E(G)} \in B^{\otimes \Phi} \cong B^{\otimes 6 n} .
$$

can be described as

$$
\left.\alpha^{\otimes E(G)}=\sum_{\operatorname{col}} \bigotimes_{(v, e) \in \Phi} \mid s_{(v, e)}=\text { color of } e\right\rangle
$$

where the sum is over all $2^{3 n}$ colorings of the edges of $G$ by 0 and 1 .

Each vertex is incident with three edges. Applying the linear map $\beta$ to each vertex and taking their tensor product, we obtain a linear map

$$
\beta^{\otimes V(G)}: B^{\otimes \Phi} \rightarrow \mathbb{R}
$$

In terms of bases, it can be described as

$$
\bigotimes_{(v, e) \in \Phi}\left|s_{(v, e)}\right\rangle \mapsto\left\{\begin{array}{cc}
1 & \text { for every } v \text { with edges } e_{1}, e_{2}, e_{3}, \text { there are } \\
& \text { exactly two } e_{i} \text { with } s_{\left(v, e_{i}\right)}=0 \\
0 & \text { otherwise. }
\end{array}\right.
$$

It follows that

$$
\beta^{\otimes V(G)}\left(\alpha^{\otimes E(G)}\right)=\operatorname{Fac}(G) .
$$

We now consider a new basis of $B$ given by

$$
|x\rangle=\frac{1}{\sqrt{2}}(|0\rangle+|1\rangle), \quad|y\rangle=\frac{1}{\sqrt{2}}(-|0\rangle+|1\rangle) .
$$

Then we can write

$$
\alpha=|x\rangle \otimes|x\rangle+|y\rangle \otimes|y\rangle
$$

with respect to this new basis. On the other hand, in this basis, values of the map $\beta$ are

$$
\begin{gathered}
\beta:|x\rangle \otimes|x\rangle \otimes|x\rangle \mapsto \frac{3}{2 \sqrt{2}}, \quad|x\rangle \otimes|x\rangle \otimes|y\rangle \mapsto-\frac{1}{2 \sqrt{2}} \\
|x\rangle \otimes|y\rangle \otimes|y\rangle \mapsto-\frac{1}{2 \sqrt{2}}, \quad|y\rangle \otimes|y\rangle \otimes|y\rangle \mapsto \frac{3}{2 \sqrt{2}}
\end{gathered}
$$


The equation (1) holds in this new basis as well. Using (2), we calculate

$$
\begin{aligned}
\beta^{\otimes V(G)}\left(\alpha^{\otimes E(G)}\right) & =\sum_{x, y \text {-col }}\left(\frac{3}{2 \sqrt{2}}\right)^{\# \text { of homogeneous vert. }}\left(\frac{-1}{2 \sqrt{2}}\right)^{\# \text { of colorful vert. }} \\
& =\left(\frac{9}{8}\right)^{n} \sum_{x, y \text {-col }}\left(-\frac{1}{3}\right)^{\# \text { of colorful vert. }} \\
& =\frac{1}{2^{3 n}} \sum_{x, y \text {-col }}(-3)^{\# \text { of homogeneous vert. }}
\end{aligned}
$$

where the sum is over all $2^{3 n}$ colorings of edges by $x$ and $y$, a homogeneous vertex is a vertex with three edges of the same color, and a colorful vertex is a vertex that is not homogeneous. This finishes the proof of Theorem 1.5 .

We note that we could have made a different change of basis in the above proof, instead of $|x\rangle$ and $|y\rangle$. The same method applied to different bases produces other similar-looking formulas. We have chosen the particular basis $|x\rangle$ and $|y\rangle$ because the end result has an particularly elegant formulation.

\section{Proof of Theorem 1.6}

It has been shown in 8 that $\Psi(r) \geq 2^{r-1}+6 \cdot 2^{\frac{r-1}{2}}-O(r)$. The graph $M C_{k}$, defined below, improves the exponential error term.

For $k \geq 3$, we define the cubic graph $M C_{k}$ as follows. There are $2 k$ vertices

$$
V\left(M C_{k}\right)=\left\{x_{i}, y_{i}: 1 \leq i \leq k\right\} .
$$

Starting with these vertices, we add the edges $x_{i} x_{i+1}$ and $y_{i} y_{i+1}$ for $1 \leq i \leq k$, so that there are two $k$-cycles. Here, the indices are taken modulo $k ; x_{i+k}=x_{i}$ and $y_{i+k}=y_{i}$. If $k$ is even, add the edges (which we colloquially call "rungs")

$$
\left\{x_{2 i-1} y_{2 i}, x_{2 i} y_{2 i-1}: 1 \leq i \leq k / 2\right\},
$$

and if $k$ is odd, add the rungs

$$
\left\{x_{2 i-1} y_{2 i}, x_{2 i} y_{2 i-1}: 1 \leq i \leq(k-1) / 2\right\} \cup\left\{x_{k} y_{k}\right\} .
$$

It is then clear that $M C_{k}$ is always a simple connected cubic graph, with cyclomatic number is given by $r\left(M C_{k}\right)=k+1$. See Figure 19 for a picture of $M_{k}$ for even $k$.

Theorem 5.1. For $k \geq 3$, the number of cycles in $M C_{k}$ is

$$
\begin{cases}2^{k}+\left(k+\frac{1}{2}\right) 2^{\frac{k}{2}}-\frac{3}{2} k & \text { if } k \text { is even, } \\ 2^{k}+\left(k+\frac{7}{2}\right) 2^{\frac{k-1}{2}}-\frac{1}{2}(3 k+5) & \text { if } k \text { is odd. }\end{cases}
$$

In particular, for $r \geq 4$,

$$
\begin{aligned}
& \Psi(r) \geq 2^{r-1}+\left(r-\frac{1}{2}\right) 2^{\frac{r-1}{2}}-\frac{3}{2}(r-1) \text { for } r \text { odd }, \\
& \Psi(r) \geq 2^{r-1}+\left(r+\frac{5}{2}\right) 2^{\frac{r-2}{2}}-\frac{1}{2}(3 r+2) \text { for } r \text { even } .
\end{aligned}
$$




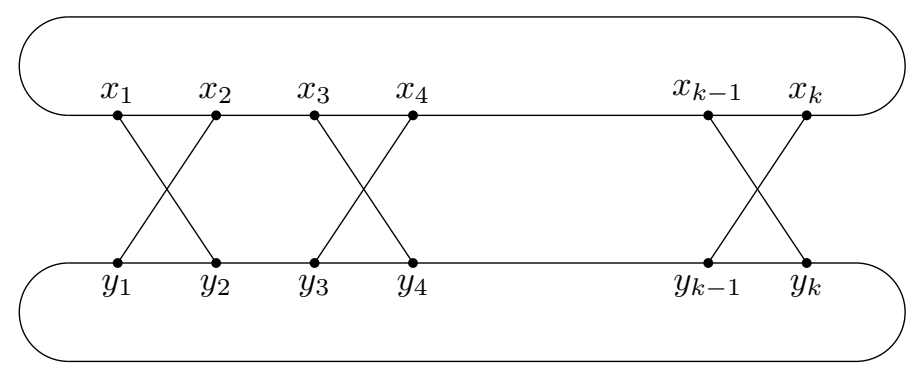

Figure 19: The graph $M C_{k}$ for even $k$

Proof. We only prove the statement for $k$ even, and leave the proof for $k$ odd to the reader. Given a cycle $C$, we first observe that the parity of

$$
w_{i}=\#\left(C \cap\left\{x_{2 i} x_{2 i+1}, y_{2 i} y_{2 i+1}\right\}\right)
$$

is independent of $1 \leq i \leq k / 2$.

We first count the number of cycles $C$ such that $w_{i}$ are all odd. Then either $x_{2 i} x_{2 i+1}$ is in $C$ and $y_{2 i} y_{2 i+1}$ is not in $C$, or $x_{2 i} x_{2 i+1}$ is not in $C$ and $y_{2 i} y_{2 i+1}$ is in $C$. There are in total $2^{k / 2}$ ways to make this choice. For each choice, there are again $2^{k / 2}$ ways of completing the set $C \cap\left\{x_{2 i} x_{2 i+1}, y_{2 i} y_{2 i+1}: 1 \leq i \leq k / 2\right\}$ to all of $C$. This shows that the total number of cycles $C$ with $w_{i}$ odd is

$$
\text { (\# of cycles } C \text { with } w_{i} \text { odd) }=2^{k / 2} \cdot 2^{k / 2}=2^{k} .
$$

We now count the number of cycles $C$ such that $w_{i}$ are all even. In this case, either $w_{i}=0$ or $w_{i}=2$. We observe that the $i$ such that $w_{i}=2$ must form an interval modulo $n$. More precisely, there exist $1 \leq s \leq n$ and $0 \leq d \leq n$ such that

$$
w_{s}=w_{s+1}=\cdots=w_{s+d-1}=1, \quad w_{s+d}=w_{s+d+1}=\cdots=w_{s+n-1}=0 .
$$

When $d=0$, the number of ways of completing to a cycle $C$ is $k / 2$, since the possible cycles are the 4 -cycles $x_{2 i-1} x_{2 i} y_{2 i-1} y_{2 i}$. If $1 \leq d \leq k / 2-1$, then for each choice of $s$ there are $2^{d+1}$ ways of completing it to a cycle. If $d=k / 2$, we only need to choose whether $x_{2 i-1} x_{2 i}$ and $y_{2 i-1} y_{2 i}$ are in $C$ or $x_{2 i-1} y_{2 i}$ and $y_{2 i-1} x_{2 i}$ are in $C$. For $C$ to be a $2 k$-cycle instead of two $k$-cycles, the number of $x_{2 i-1} y_{2 i}$ in $C$ must be odd, hence there are $2^{\frac{k}{2}-1}$ cycles. Then

$\left(\#\right.$ of cycles $C$ with $w_{i}$ even $)=\frac{k}{2}+\sum_{d=1}^{n-1} n \cdot 2^{d+1}+2^{\frac{k}{2}-1}=\frac{k}{2} \cdot\left(2^{\frac{k}{2}+1}-3\right)+2^{\frac{k}{2}-1}$

Hence, in aggregate, the number of cycles in $M C_{k}$ is

$$
2^{k}+\left(\frac{k}{2}+\frac{1}{4}\right) 2^{\frac{k}{2}+1}-\frac{3}{2} k .
$$

The stated lower bound on $\Psi(r)$ follows directly from $r\left(M C_{k}\right)=k+1$. 


\section{Acknowledgment}

The authors are grateful to Noga Alon for discussions on the main result of this paper.

\section{References}

[1] W. Ahrens. Über das Gleichungssystem einer Kirchhoff'schen galvanischen Stromverzweigung. Math. Ann., 49(2):311-324, 1897.

[2] R. E. L. Aldred and Carsten Thomassen. On the maximum number of cycles in a planar graph. J. Graph Theory, 57(3):255-264, 2008.

[3] Noga Alon and Shmuel Friedland. The maximum number of perfect matchings in graphs with a given degree sequence. Electron. J. Combin., 15(1):Note $13,2,2008$.

[4] L. M. Brègman. Certain properties of nonnegative matrices and their permanents. Dokl. Akad. Nauk SSSR, 211:27-30, 1973.

[5] R. C. Entringer and P. J. Slater. On the maximum number of cycles in a graph. Ars Combin., 11:289-294, 1981.

[6] Louis Esperet, František Kardoš, Andrew D. King, Daniel Král, and Serguei Norine. Exponentially many perfect matchings in cubic graphs. Adv. Math., 227(4):1646-1664, 2011.

[7] G. Galbiati. An exact upper bound to the maximum number of perfect matchings in cubic pseudographs. Calcolo, 18(4):361-370 (1982), 1981.

[8] Peter Horak. On graphs with many cycles. Discrete Math., 331:1-2, 2014.

[9] László Lovász and Michael D. Plummer. Matching theory. AMS Chelsea Publishing, Providence, RI, 2009. Corrected reprint of the 1986 original [MR0859549]. 